\title{
Removal of Dye by Biological Methods Using Fungi
}

\author{
Behnaz Abbasi ${ }^{1, *}$ \\ ${ }^{1}$ Department of Microbiology, Falavarjan Branch, Islamic Azad University, Isfahan, Iran
}

Corresponding Author: Behnaz Abbasi, MSc, Department of Microbiology, Falavarjan Branch, Islamic Azad University, Isfahan, Iran. E-mail: abbasi.behbanz@gmail.com

Received October 22, 2017; Accepted November 22, 2017; Online Published December 25, 2017

\begin{abstract}
Synthetic dyes are increasingly used in industries such as medicine, pharmaceuticals, textiles, paper, cosmetics, leather, photography, food, etc. These compounds have severe environmental effects; most are toxic, mutagenic, and carcinogenic. In addition, they are usually resistant to degradation, and decolorizing them by physical or chemical methods, such as surface absorption, sedimentation, chemical analysis, light analysis, etc., requires substantial amounts of money, time, and energy. At the moment, the attention of most researchers in this field is focused on microorganisms with the ability to remove dyes from color materials. Innovative processes for the treatment of industrial wastewater which contains heavy metals and dyes are often used to reduce environmental pollution and the toxicity of these pollutants to cause the wastewater to meet purification standards. Research has shown that microorganisms such as bacteria, fungi, and yeasts can play an important role in decolorizing wastewater. The purpose of this paper is to review the causes of contamination and the role of fungi in decolorizing industrial wastewater.

Keywords: Decolorization; Fungi; Waste Water

Citation: Abbasi B. Removal of dye by biological methods using fungi. Int J Med Rev. 2017;4(4):112-118. doi: 10.29252/ijmr040405.
\end{abstract}

\begin{abstract}
Introduction
Population growth, urbanization, increased metal extraction, industrial progress, and the production of new compounds are causing a more polluted environment. Pouring treated or untreated industrial and household wastewater into the environment has contaminated the shores of lakes and rivers with pharmaceuticals, oil products, heavy metals, colors, toxins, and various chemical compounds. Neither chemical nor physical methods of pollutant removal have been able to properly solve this environmental problem. In addition, the increasing volume of water used in various industries, including textiles, paper, plastics, and dyestuffs, along with other chemicals known as carcinogens, like organic dye materials on the one hand, and the waste of $10 \%-15 \%$ of these chemicals on the other have produced a high volume of colored wastewaters that cannot be directly released into the environment. ${ }^{1}$ Looking at the systemic law of nature, we find that natural cycles act to create safe and clean substances for the environment. Thus, working on new methods to remove materials is important. Dyes are one of the most important pollutants of wastewater from the dyeing industry and include organic substances with complex, often toxic, mutagenic, and non-biodegradable
\end{abstract}

structures. ${ }^{2}$ Dyestuffs absorb light on the water surface and prevent the growth of aquatics in the water, which can have harmful effects on the food chain. Also, high stability and water solubility, two basic advantages for the production sector, are considered two negative factors in the environment as they increase the hazards of using these materials. Among dyes, Azo dyes comprise the most important commercial class of colors, accounting for more than $50 \%$ of the total dyes produced worldwide. For this reason, different purification systems are used to remove these materials from industrial wastewaters. Different methods are used for dye removal, such as physical, chemical, biological, and advanced ones. Among these methods, if physical methods are effective, they can be considered cost effective, because chemicals are not used and intermediate products are not produced. Of course, in a refinery, physical refinement alone is not enough to dispose of sewage pollution, and it is better to use biorefinement in addition to mechanical methods. This type of refinement is called secondary refining. ${ }^{3}$ The importance of this method is especially evident in lowconcentration wastewaters, where the use of chemical methods is not cost effective, ${ }^{4}$ because in biological

Copyright $(02017$ The Author(s). This is an open-access article distributed under the terms of the Creative Commons Attribution License (http://creativecommons.org/licenses/by/4.0), which permits unrestricted use, distribution, and reproduction in any medium, provided the original work is properly cited. 
processes, the hazardous wastewater is purified using living and non-living organisms to break or degrade pollutants into non-toxic or less toxic materials. ${ }^{5,6}$ On the other hand, the absorption method, widely used as a physical process in most pre-refinement sectors, is associated with the expansion of a variety of absorbents, including mineral absorbents such as zeolite, perlite, kaolin, and carbon dioxide, ${ }^{7}$ and even polymeric absorbents, ${ }^{8}$ but with the benefits of a biological system, if these post-mortem biocompounds could be used as absorbents. In addition, the cost of their refinement process can be greatly reduced due to their relatively low cost. Recent studies have shown that different fungi and algae have significant absorption capacities for the removal of heavy metals, ${ }^{9,10}$ but few studies have focused on removing dyes from colored effluents. Some studies have mentioned, for example, the absorption of active ${ }^{11}$ and direct dyes ${ }^{12}$ by Aspergillus niger. Therefore, in this study, the effects of biological agents, especially fungi, on biological decolorization have been studied.

\section{Industrial Dyes}

Colors are one of the most important categories of water pollutants and only one entry into water is enough to reduce water quality significantly. Due to the synthetic origin and presence of complex molecules in the structure of dyes, the purification process will be accompanied by problems in some cases. ${ }^{13}$ The wastewater of many industries, such as textile, dyeing, and paper industries, contains a variety of colors that, in addition to ruining the appearance of water resources, prevent the penetration of light into the depths of the receiving waters. Due to the high COD content of colored wastewater, their evacuation reduces the oxygen dissolved in water and threatens the life of aquatic animals. Many dye combinations contain heavy metals and hazardous organic substances, and by remaining in water resources, they present a serious threat to human health and the environment. In the following, we refer to a variety of contaminating colors.

Dyes in the Leather Industry

In the leather industry, the trivalent chromium salts used in tannin under acidic conditions, such as chromic sulfate, produce blue wastewater resulting from a trivalent chromium cation. Therefore, this effluent contains not only color, but also great amounts of chromium. Under oxidative conditions it can be converted to hexavalent chromium, which is hazardous and toxic to human beings. Trivalent chromium is the essential chromium in human body with no systemic effects reported ${ }^{14}$. Watering agricultural lands with chrome-contaminated wastewater increases its concentration in agricultural products, which can cause diseases.

Azo Dyes

Azo dyes comprise the largest and most diverse group of synthetic colors. They are widely used in various industries, including the textile, food, cosmetics, paper, and other industries, accounting for $70 \%$ of the total amount of color used worldwide. In theory, azo dyes can create a wide range of colors, but in commerce, they provide yellow, orange, and red colors more than others. Azo dyes, compared to other common color groups, such as anthraquinone, produce brighter and more intense colors, but are weaker than carbonyl and phthalocyanine groups. The greatest advantage of azo dyes is their low cost. Azo pigments are colorless particles that are painted using a combination of azo compounds. Azo pigments are important in some colors, like in art supplies. They have excellent color properties, especially in the yellow and red spectra, and have a good color stability against sunlight. This color stability depends not only on the properties of the organic azo compound, but also on how it is absorbed into the pigment carrier. Different types of these colors which have carboxylic and sulfonic groups can be used in many fields, but the main disadvantage of using these colors is their biological degradation. For example, textile industries produce a large amount of wastewater with many contaminants, including phenols, sulfurs, chromium, and dyes. Colored wastewaters create not only eyesight problems caused by intense color, but may also cause environmental toxicity, if released in the environment without any treatment. These colors are categorized in terms of the number of azo groups into monoazo, diazo, and polyazo. Monoazo dyes have one group of azo and are the most commonly used azo groups. These colors are divided into two groups: colors without carboxylic and sulfonic groups, and colors with acidic groups. ${ }^{15}$ Diazo dyes have two groups of azo. In the structure of these colors, coupling material is coupled on both sides by two diazonium groups. The number of these colors is limited. They are often insoluble in water and are practically considered as dentate and acidic dyes. One of the most important groups of these colors is black acid. The coupling substance in tetraazonium dyes is coupled with a tetraazonium compound. One of the most important of these colors is Red Congo, which is made up of a pair of benzidine with two groups of nefitonic acid. These colors include pigments, direct colors, and a number of acidic and dentate colors. The D substance in the combination of these colors determines the type of acidic or dentate color. Diamines such as benzidine are an example of this compound. The Red Congo is considered one of the direct colors of this group and is used for dyeing polyamide, wool, and cellulose fibers. Due to insufficient stability, they are commonly used in titrations as reagents. This color is also used in the food industry. Azo dyes are much more stable than most natural dyes. These colors are stable in the whole $\mathrm{pH}$ range in foodstuffs, are heat resistant, and do not lose color in light or oxygen. Thus, they can be used in all foods. Of course, one disadvantage of these colors is their insolubility in oils or fats. Azo dyes only color oil if they are coupled with a fat molecule or dispersed in oil as very fine particles. Azo dyes do not have a very intense 
toxicity. Because of their high coloring power, low amounts of color are enough to create the desired color in foods, and since azo dyes are highly soluble in water, they never accumulate in the body, are metabolized in the liver, and are excreted in urea. In addition to the above, some of them cause genetic mutations and are carcinogens. If these colors can be removed properly from wastewater, they can be used in various industries. ${ }^{16}$

\section{Biologic Decolorization}

Today, the removal of colors and pigments from wastewater is widely studied, because public concerns are increasing due to the pollution of sewage by dyes. The general disregard for the presence of colors in water, even at low concentrations, is the main reason for this. In addition, several groups of colors are stable molecules, resistant to decomposition by light, chemical, biological, or other factors, and are considered mutagenic to humans. ${ }^{17}$ The problem of colored wastewaters started from artificial dyes. It is estimated that annually, more than 28,000 tons of textile dye enter the environment from various industries. Azo dyes make up $70 \%$ of the weight of the dyes used in the world, making it the largest and most consumable artificial dye entering the environment. Most colors are complex organic molecules and require resistance to external factors such as detergents. Color molecules consist of two components: a color agent responsible for the formation of color and a reinforcement factor responsible for enhancing the color factor, solubility in water, and bonding to the surface. Therefore, it is essential that the contaminated wastewater be treated with suitable methods prior to being released into the environment. The release of untreated or incompletely treated wastewater from industries and households to the environment and into water sources has contaminated the shores of lakes and rivers with pharmaceuticals, oils, heavy metals, colors, toxins, and various chemical compounds. Neither chemical nor physical methods for removing pollutants have been able to properly solve this environmental problem. Looking at the systemic law of nature, we find that natural cycles act to create safe and clean substances for the environment. Thus, working on new methods to remove materials is important. One of these methods is the biological removal of pollutants, or biotechnology in removing pollutants, including the use of plants, various microorganisms such as bacteria, fungi, or yeasts, or parts of plants or animals. On the other hand, the mass production of these microorganisms is much easier than other methods. There are a variety of fungi that can remove color, like string-shaped soil fungi such as Fusarium and white rot fungi such as Hypoxylon, Ketomium, Pyulospora, Coriolis, Polaria and Fenorocyte. For example, in 1985, Aspergillus orisa and Aspergillus fumigatus were used to decolorize textile waste. Aspergillus niger and Rayzor Orisa have been used for decolorizing textile wastewater. ${ }^{18}$ According to the above, biologic decolorization can be the best and most cost-effective method. This study examines decolorization by fungi as follows.

Decolorization by Fungi in the Leather Industry

Recent studies have shown that fungi can be effective in removing color, heavy metals, organic substances, and toxic compounds. ${ }^{19}$ Considering the fact that using fungi in decolorizing dye from the effluent of the leather industry has not been accurately studied and that the efficiency of Aspergillus niger has been proven in decolorizing other industrial waste, the extent of decolorizing this fungus can perform from tannin waste, which has always been problematic when disposed into the environment, was investigated.

\section{Decolorization in Textile Industries}

For the decolorization of textile wastewater, several methods, including surface absorption, filtration, coagulation, chemical decolorization, photocatalytic decolorization, and decolorization with ozone, have been used. The removal of color by biological absorption has also been suggested. Most of these methods are expensive and costly, even if they produce desirable results. The use of biological processes is another method proposed for textile wastewater treatment. However, it should be noted that textile colors have a synthetic origin and are composed of complex aromatic molecules designed to resist discoloration and biological erosion, so it is not easy to decompose and remove color through biological processes. Nonetheless, compared to physical and chemical methods, the biodegradation of azo dyes has been considered more, because this method has lower costs and produces fewer toxic substances. In many microorganisms, like bacteria, fungi, and even molds, the decolorization and decomposability properties of azo dyes have been observed. Among these microorganisms, bacteria and fungi play a key role in the treatment of colored wastewater even in the presence of various enzymes. Of course, it should be noted that although microbial methods require less energy, due to the specific characteristics of microorganisms, their application also has some problems, including the fact that they are pathogenic for plants, have a restricted substrate, or have low growth speed. Various methods have been proposed for decolorization by bacteria, most of which often result in the production of aniline, which is itself a toxic substance. Therefore, bacteria are not suitable for the decomposition of these colors, because they produce toxic substances and cause environmental pollution. Using fungi for decolorization is newly proposed to result in the decomposition of colors without producing toxic substances. In this regard, it is necessary to use one fungus or a set of fungi together to perform the biological degradation of the color. So far, there are more reports on the capacity of white rot fungi, especially phanerochaete chrysosporium, to decompose azo dyes than others. White rot fungi are known for their 
potential ability in the production of extracellular oxidizing enzymes. It has been shown that some of the ligninolytic extracellular enzymes produced by these fungi, including lignin peroxidase (Lip), manganese peroxidase (Mnp), horseradish peroxidase (Hrp), and laccase, are effective in decomposing azo dyes. The extracellular enzymatic system enables the fungus to tolerate high concentrations of pollutants. Also, reports have been provided on the decolorization of azo dyes by the penicillium genus, though its decolorization mechanism is still unknown. ${ }^{20}$ In 2007 , research was conducted on the decolorization and toxicity of industrial reactive colors using Trametes pubescens and Pleurotus ostreatus fungi. The two fungi were fixed in a substrate on polyurethane cubes inside a bioreactor.

In this study, five consecutive cycles were performed for each color and fungus. Activity of laccase peroxidases, dependent and non-dependent to $\mathrm{MN}$, lignin peroxidase, and aryl-alcohol oxidase were recorded during each cycle. The toxicity of each color environment was determined by the Lemna minor (duckweed) ecotoxicity test. The study found that both fungi have the ability to decolorize all colors, even at the highest concentrations, and they significantly reduced the toxicity of wastewater. T. pubescens showed higher detoxification and decolorization ability than P. ostretus. Other types of fungi used in the decolorization of textile wastewaters include Aspergillus fumigatus and A. niger. In a study at the Biotechnology Research Institute of Iran's Industrial Research Organization, Aspergillus niger could remove $98 \%$ of the textile dyes in wastewater within 18 hours. $^{21}$

\section{Decolorization in Pulp Industry}

A significant number of fungi have been identified as human pathogens, and a larger group is also known as being pathogenic for plants. These creatures have always been counted among the most important wood destructors, as they cause great damage to wood each year, resulting in loads of rotten wood. Wood-decaying fungi are the first living wood destructors. In the forest ecosystem, they have the most important role in the carbon and nitrogen cycle, and they help the organic conversion of wood chips and particles into the soil's humus layer. Except for a few ascomycota fungi, wooddecaying fungi are mainly basidiomycete and dependent on Agaricales, Hymenochaetales, Polyporales and Russulales. ${ }^{22}$ White rot fungi are primarily considered for use in paper-making processes. Although most of these fungi have the potential to destroy lignin, some of them also destroy cellulose and hemicellulose. ${ }^{23}$ White rot fungi can destroy more lignin than other cellular wall elements. They selectively degrade lignin, and this is what the paper-making processes are pursuing. These fungi are also the focus of research in biological fermentation. ${ }^{24}$ Considering that the implementation of pollution reducing programs in the pulp and paper industry is generally expensive and requires costly operations, researchers have conducted many studies on the biological decolorization of paper pulp. The concept of biological pulping was probably obvious for primary researchers. Perhaps the first serious work in this field was carried out in the 1950s by researchers at the Vesta Virginia Pulp and Paper Laboratory. Their research led to the publication of an article. They investigated 72 types of lignin-destroying fungi in this study. ${ }^{25}$ The investigation of the natural degradation of pine wood by white rot fungi for the chemical pulp industry showed that most increased the paper resistivity properties. ${ }^{26}$ Types of white rot fungi that selectively reduce the amount of lignin are useful for the biologic pulp industry. Ideal systems for biologic pulp must have the potential to reduce environmental impacts and energy consumption. The most effective species found in fungi useful for wood include ceriporiopsis subvermispora, phlebia brevispora, phanerochaete chrysosporium. ${ }^{27}$ The potential of different fungi for use in pulping has been studied in different parts of the world.

The results of these studies showed that the appropriate fungus for this purpose should have two characteristics: 1) high extent of destruction, and 2) the potential to degrade lignin. According to the results of these studies, white rot fungi, from the Basidiomycete species, have been identified as the preferred option in this regard. ${ }^{28-30}$ The ability of four fungi, Coriolus versicolor, Phanerochaete charysosporium, IZU-154, and Coriolus hirsutus, was tested in broad-leaf craft pulp. ${ }^{31}$ The fungus IZU-154 produced the greatest amount of white color. They also used the needle-leaf craft pulp for biological decolorization by IZU-154 white rot fungi and observed that the glow of the craft pulp increased. Sakai et al. showed that the fungus Phanerochaete sordida yk-624, separated from rotten wood, had strong activity in reducing lignin and decolorizing the craft pulp ${ }^{32}$ as well as increasing the pulp glow. In their research, Scott et al. concluded that the treatment of wood chips with lignindestroying fungi improves paper resistance. ${ }^{33}$ Wolfart et al. found that fungal treatments with Phanerochaete charysosporium enhance the resistive properties of the resulting paper. Various studies have also been carried out on various types of white rot fungi to find the most suitable one for biological pretreatment. Based on the amount of chemical agents used, the reduction of kappa coefficient, the improvement of the resistive properties of the resulting paper, and the reduction of environmental pollution during the chemical process, the fungus of the Ciroporiopsis subvermispora series was introduced as the most suitable one. ${ }^{29,34}$ Hernandez et al. showed that 2 weeks of pretreatment with the fungus on Noelle wood for mechanical pulping significantly increased paper resistance, including tear length and stretch. Fungi that cause white rot (basidiomycetes) grow inside cellular wood cavities and attack all cell wall components from the internal parts of the cell cavity. ${ }^{35}$ Some attack cellulose and lignin, and others use the entire cell wall component at a similar degree. During the 
activity of white rot fungi, wood is converted into a mass of white warps and filaments, and its weight may eventually exceed $95 \%$. This kind of decay is often specific to broad-leaves. Evidence available so far suggests that three enzymes are the main causes of lignin polymer degradation and the production of low molecular weight materials in white rot fungi. These enzymes include lignin peroxidase, manganese peroxidase, and laccase. Some fungi produce all three enzymes, but this is not common in all fungi. ${ }^{36}$

The ability of three white rot fungi, Ceriporiopsis subvermispora, Phanerochaete charysosporium and Trametes versicolor, to change and degrade lignin during cooking and decolorizing were studied on two species of acacia and eucalyptus for three periods of two weeks for wood and paper pulp. The results showed that the properties of handmade paper significantly improved with pre-treatment of fungi before decolorization. Also, due to the properties of pulping and paper making, the effectiveness of C. subvermispora was more favorable than that of the other two fungi. ${ }^{37}$ During a research on C. subvermispora, it was found that this fungus could destroy unsaturated fatty acids under biopulping conditions. Using UV spectrophotometer and gel chromatography to analyze lignin compounds in the destroyed wood indicated that fewer soluble lignin compounds in the grounded wood were reduced. In another study, a combination of broadleaf wood was treated with lignin-destroying fungi, and the effect of fungal pre-treatment on the decolorization properties of craft pulp was investigated. ${ }^{38}$ In the pretreated wood chips, the amount of active alkali was reduced in comparison with the non-treated samples. The obtained results showed that alkalinity was decreased in the treated wood chips and was increased in the non-treated samples. Fungal treatments produced effective energy during the process and consumed about $4.4 \%$ less chlorine compared to the control sample. Pre-treatment of samples with Ceriporiopsis subvermispora caused a reduction of $7.4 \%$ lignin and $3.14 \%$ of potassium permanganate (kappa coefficient).

\section{Decolorization by Laccase Enzyme Derived from Fungi}

One major biomass compound is lignocellulose, which contains about half of the substance resulting from the photosynthetic process. Lignocellulose is composed of three types of cellulose, polymer, hemicellulose, and lignin, which chemically bond to each other by noncovalence and cross-linking covalent bonds. Some microorganisms, especially fungi types, have developed certain enzymatic systems to obtain the ability to degrade lignin. These fungi are divided into three types of rot according to their shapes: white, brown, and soft rot. The reactions of fungi, in particular white rot fungi, to affect lignin are often carried out by a series of extracellular peroxide enzymes called ligninases, which include laccase (Lac: EC 1.10.3.2), lignin peroxidase (Lip: EC
1.11.1.14), manganese peroxidase (Mnp: EC 1.11.1.13), and others. Among the ligninolynic enzymes, phenol oxidases called laccase and peroxides, such as lignin peroxidase and manganese peroxidase, play a major role in lignin decomposition. Benzodiazole laccase, oxygen oxidoreductase (EC: 1.10.3.2) are a group of enzymes called blue copper oxidases. Laccase uses oxygen as an electron receptor and produces quinone; therefore, it is part of the phenol oxidase family. This enzyme oxidizes a range of organic substrates including mono-, di-, and poly-phenols, aromatic amines, carboxylic acids, and non-phenol and inorganic substrates. Of course, it tends to oxidize para-diphenol more. This enzyme, due to the oxidative power of phenolic and non-phenolic lignin compounds, can degrade similar compounds that are hardly degradable in nature and contribute to the removal of pollutants. Laccase is widely distributed in nature, found in many organic plants, fungi, bacteria, and even some insects. Laccases are found in a variety of fungi, including ascomycetes, deuteromycetes, and in particular in a number of basidiomycetes known as white rot fungus. The laccase producers in Ascomycota fungi include Gaeumannomyces graminis Melanocarpus albomyces, Monocillium, Neurospora crassa, Podospora anserina, Aspergillus, Curvularia, and Penicillium. Considering the importance of the laccase enzyme in biotechnology and its wide use in industry, extensive research is currently being carried out around the world to isolate types of good microorganisms that produce this enzyme for use in industry. This enzyme can be an alternative to other common oxidants (such as sodium hypochlorite). Laccase has the ability to decolorize indigo and can achieve a wide variety of effects. Laccase alone does not have the ability to decolorize indigo; when combined with an appropriate mediator compound, it can acquire the quick decolorization ability. Oxidation is carried out by transferring an electron from the donor to the receiving substance. Here, the electron is transmitted from indigo to oxygen by an enzyme and a mediator compound. The mediator is a low molecular weight organic compound. The bond between the two carbonyl groups is broken down in indigo and coloring chromophores are destroyed. As a result, indigo is oxidized and the red combination of Nile and oxygen is achieved. Laccase does not have the ability to oxidize black sulfur dye, which is mainly used on denim. The laccase reaction stops when the active mediator agent is finished. Therefore, the decolorization amount can be controlled correctly by carefully selecting the amount of laccase and time. In this way, there will be fewer environmental problems than with sodium hypochlorite. Advantages of this method include minimal reduction of strength, reduced time, repeatability (as controlling the process is more convenient), reduction of water consumption, and greater safety than other decolorization methods. The original commercial product, produced in 1996, was a liquid slurry. This product had a good performance, but its properties were 
not ideal when tested. A commercial solid form of laccase has been commercially available since 1999 for denim. The latest commercial product consists of a laccase enzyme, mediator, buffer, and crushed components. Today, laccase formula is based on different needs and used for garment washing processes. ${ }^{39}$

\section{Conclusions}

Environmental pollution and health hazards caused by colored materials are widely produced by various industries. The stability of these materials makes it possible to examine various methods for their removal. The decolorization of industrial wastewater is one of the most important environmental concerns. There are various ways to eliminate this type of contamination, but for reasons such as not being economical and requiring long periods of time, the best solution for decolorization is the biologic method. Among microorganisms, such as bacteria or fungi, it was found that fungi can play an effective role in decolorizing the wastewater of textile, leather, and paper-making industries and can be used for a variety of reasons such as cost-effectiveness, easy access, environmental compatibility, and shorter time frame than other methods. Therefore, given the sensitive environmental conditions of using fungi, priority should be given to new methods of decolorization.

\section{Conflict of Interest Disclosures}

None.

\section{Funding}

None.

\section{References}

1. Qu B, Zhou J, Xiang X, Zheng C, Zhao H, Zhou X. Adsorption behavior of azo dye C. I. acid red 14 in aqueous solution on surface soils. J Environ Sci (China). 2008;20(6):704-9. pmid: 18763565.

2. Qani Zadeh G, Asgari G. [Methylene blue dye removal from wastewater using bone ash]. J Hamedan Univ Med Sci. 2009;2(2):104-13.

3. Chatha SAS, Asgher M, Ali S, Hussain Al. Biological color stripping: A novel technology for removal of dye from cellulose fibers. Carbohydrate Polymers. 2012;87(2):147681. doi: 10.1016/j.carbp ol.2011.09.041.

4. Roys N. Optimal investment policy with fixed adjustment costs and complete irreversibility. Econom Let. 2014;124(3):416-9. doi: 10.1016/j.econ let.2014.06.026.

5. Qian J, Lu H, Cui Y, Wei L, Liu R, Chen GH. Investigation on thiosulfate-involved organics and nitrogen removal by a sulfur cycle-based biological wastewater treatment process. Water Res. 2015;69:295-306. doi: 10.1016/j.watres.2014. 11.0 38. pmid: 25497428.

6. Cullough MC, Hazon J. Introduction to bioremediation. USA: Department of energy NABIR grant; 2002.

7. Bahmaei M, Rahnavard Kissomi Z, Olya M, Kasehgari $\mathrm{H}$. Removal Of Labs Anionic Surfactant By Perlite And Activated Carbon. J Appl Res Chem. 2013;7(2):67-73.

8. Pirkarami A, Olya ME, Najafi F. Removal of azo dye from aqueous solution using an anionic polymeric urethane absorbent (APUA). J Ind Eng Chem. 2015;21:387-93. doi: 10.1016/j.jiec.2014.02.050.

9. Çetinkaya Dönmez G, Aksu Z, Öztürk A, Kutsal T. A comparative study on heavy metal biosorption characteristics of some algae. Proc Biochem. 1999;34(9):885-92. doi: 10.1016/s0032-9592(99)00005-9.

10. Nuhoglu Y, Malkoc E, Gurses A, Canpolat N. The removal of $\mathrm{Cu}(\mathrm{II})$ from aqueous solutions by Ulothrix zonata. Bioresour Technol. 2002;85(3):331-3. doi: 10.1016/s09608524(02)00098-6. pmid: 12365504.

11. Khalaf MA. Biosorption of reactive dye from textile wastewater by non-viable biomass of Aspergillus niger and Spirogyra sp. Bioresour Technol. 2008;99(14):6631-4. doi: 10.1016/j.biorte ch.2007.12.010. pmid: 18242981.

12. Abd El-Rahim WM, El-Ardy OAM, Mohammad FHA. The effect of $\mathrm{pH}$ on bioremediation potential for the removal of direct violet textile dye by Aspergillus niger. Desalination. 2009;249(3):1206-11. doi: 10.1016/j.desal.2009.06.037.

13. Forgacs $E$, Cserhati T, Oros G. Removal of synthetic dyes from wastewaters: a review. Environ Int. 2004;30(7):953-71. doi: 10.1016/j.envint.2004 .02.001. pmid: 15196844.

14. Noori S, Rahnama M. [The quality of drinking water]. Semnan: Medical university of Semnan publication; 1995.

15. Toh Y-C, Yen JJL, Obbard JP, Ting Y-P. Decolourisation of azo dyes by white-rot fungi (WRF) isolated in Singapore. Enzyme Microb Technol. 2003;33(5):569-75. doi: 10.1016/s0141-0229(03)00 177-7.

16. Martins MAM, Lima N, Silvestre AJD, Queiroz MJ. Comparative studies of fungal degradation of single or mixed bioaccessible reactive azo dyes. Chemosphere. 2003;52(6):967-73. doi: 10.1016/s0045-6535(03)00286-8.

17. Crini G. Non-conventional low-cost adsorbents for dye removal: a review. Bioresour Technol. 2006;97(9):1061-85. doi: 10.1016/j.bior tech.2005.05.001. pmid: 15993052.

18. Mazaheri Tehrani M. [Decontamination of wastewater from textile factories by fungi and appropriate strain selection]. Tehran: University of Tehran; 1997.

19. Griffin D. Spore dormancy and germination. Fungal physiology. 2nd ed. New York, NY: John Wiley \& Sons; 1994. p. 375-98.

20. Yang Q, Li C, Li H, Li Y, Yu N. Degradation of synthetic reactive azo dyes and treatment of textile wastewater by a fungi consortium reactor. Biochem Eng J. 2009;43(3):22530. doi: 10.1016/j.bej.2008. 10.002.

21. Jahangir M. [The effect of different factors on the growth of the marine strain to remove the color]. 4th Iranian Chemical Engineering Congress; Iran1998.

22. Gonthier P, Nicolotti G. A field key to identify common wood decay fungal species on standing trees. Arboricult Urb Forest. 2007;33(6):410.

23. Blanchette RA, Behrendt CD, Williams D, Iverson $S$, Akhtar $M$, Enebak SA, editors. New approach to effective biopulping: Treating logs with Phlebiopsis gigantea. Proceedings of the 1998 7th International Conference on Biotechnology in the Pulp and Paper Industry Part 1 (of 3); 1998: CPPA.

24. Ebrahim Poor G, Homsi M. [Effect of fungal treatment on resistance properties of chemical-mechanical pulp of hornbeam wood]. Iranian Q J Sci Res Wood Paper 2012;7(1):1-15

25. Lawson Jr L, Still C. The biological decomposition of ligninliterature survey. Tappi J. 1957;40(9):56A-80A.

26. Kawase K. Chemical components of wood decayed under natural condition and their properties. J Fac Agricult Hokkaido Univ. 1962;52(2):186-245.

27. Villalba LL, Scott GM, Schroeder LR. Modification of Loblolly Pine Chips withCeriporiopsis subvermisporaPart 2: Kraft Pulping of Treated Chips. J Wood Chem Technol. 2006;26(4):349-62. doi: 10.1080/02773810601105 185.

28. Fillat Latorre A. Flax fibre modification using enzyme systems to obtain high-value cellulose products. Catalunya: Universitat Politècnica de Catalunya; 2011.

29. Kirk T, Koning J, Burgess R, Akhtar M, Blanchette R. Biopulping: A glimpse of the future. Forest Service research paper. Forest Service, Madison, WI: Forest Products Lab; 1993. 
30. Wolfaardt JF. Utilization of wood-decay fungi for biokraft pulping of softwood 1999.

31. Fujita K. Biobleaching of kraft pulp using white-rot fungus IZU-154. Tappi J. 1991;74:123-7.

32. Bajpai P, Kondo R. Biotechnology for environmental protection in the pulp and paper industry: Springer Science \& Business Media; 2012.

33. Scott GM, Swaney R. New technology for papermaking: biopulping economics. Tappi J. 1998;81(12):153-7.

34. Akhtar M, Attridge M, Blanchette R, Myers G, Wall M, Sykes $M$, et al. The white-rot fungus Ceriporiopsis subvermispora saves electrical energy and improves strength properties during biomechanical pulping of wood. Biotechnol Pulp Paper Ind. 1992:3-8.

35. Hernández M, Hernández-Coronado MJ, Pérez MI, Revilla E, Villar JC, Ball AS, et al. Biomechanical pulping of spruce wood chips with Streptomyces cyaneus CECT 3335 and handsheet characterization. Holzforschung. 2005;59(2). doi: 10.1515/hf.2005.027.

36. Schmidt O, Czeschlik D. Wood and tree fungi. Berlin: Springer-Verlag; 2006.

37. Malinen R, Islam M, Karim M, editors. Beneficial Effects of Fungal Treatment before Pulping and Bleaching of Acacia mangium and Eucalyptus Camaldulensis. 59th Appita Annual Conference and Exhibition; 2005; Auckland, New Zealand.

38. Rajabi S, Afraa A. [Investigating the process of biological pulping and its performance on reducing wastewater in the stage]. 2002.

39. Madhavi V, Lele S. Laccase: properties and applications. BioResources. 2009;4(4):1694-717. 